H. C. Jones ${ }^{1}$ attempts to explain the effect on the basis of the solvate theory. In his explanation the transmission of the water should be increased in all the absorption bands by the hydrating substances, and should be unaffected by the non-hydrating substances. This explanation cannot fit the present results without some serious modifications.

CORNELl UNIVERSity, December, I92I.

The Absorption Spectrum of Hydrogen Chloride at $200^{\circ} \mathrm{K}$.

By B. J. Spence and C. Holley.

PlANCK ${ }^{2}$ assuming that Bohr's static orbits are not the only possible ones (an assumption compatible with the classical laws of electrodynamics) has made a study of the absorption spectrum' due to the rotations of electrical doublets with free axes. He finds as one result of this investigation that maximum absorption should occur at the wave length

$$
\lambda \max =2 \pi c \sqrt{\frac{J}{K T}},
$$

where $c$ is a constant, $K$ Planck's constant, $J$ the moment of inertia of the doublet, and $T$ the absolute temperature.

The investigation is an attempt to verify the above formula. The absorption of the diatomic gas hydrogen chloride was studied at room temperature and also at $200^{\circ} \mathrm{K}$., just above its liquefaction temperature $\left(-83^{\circ} \mathrm{C}\right.$.) at atmospheric pressure. This gas shows absorption bands beyond Io $\mu$ which are attributed to molecular rotation. It also shows a series of bands in the region of $3.5 \mu$ which according to Bjerrum (Nernst Festschrift, I9I2) are due to the combination of the frequencies of the bands beyond Io $\mu$ with that of the vibration of the molecule. In the application of the Planck expression, we have assumed that any variation in absorption beyond Io $\mu$ will make itself evident as a change in the absorption in the region at $3.5 \mu$. to which we have confined our study. The investigation was carried on by means of an infra-red spectroscope of the fixed arm type, using a plane grating of 2,500 lines per inch, kindly loaned to us by Professor H. M. Randall of the University of Michigan.

It was found that there was a greater general absorption at the low temperature than at room temperature. In addition, it was found that the bands due to the lower rotational frequencies as evidenced in the $3.5 \mu$ region were very much less pronounced, almost disappearing at the low temperature, while those corresponding to the higher frequencies were not diminished in anything like a similar ratio. It was also found that the number of bands observed remained the same, with no shift in their wave-length at the low temperature.

${ }^{1}$ Phys. Zeit., Vol. I5, p. 447, I9I4.

2 Annalen der Physik, 53, 24, I9I 7. 
At the low temperature we expected on the basis of the Planck expression that the bands due to the higher frequencies of rotation would be less pronounced or decreased in a greater ratio than those due to the lower frequencies. The investigation appears not to confirm the Planck expression for the temperature chosen.

NORTHWESTERN UNIVERSITY.

\section{Two New Lines in the Aluminum Spectrum and Their Possible Series Relations.}

By G. D. Shallenberger.

WHEN a high potential vacuum spark, which has been described by Millikan, ${ }^{1}$ is applied to $\mathrm{Al}$ electrodes, two unidentified lines are obtained in the spectrum of this element. They are at $\lambda_{4150.6}$ and $\lambda 2907.4$ respectively. Both are strong, well-defined lines. The one with the longer wave-length has the higher intensity rating. It is of about the second or third order of magnitude, while the shorter line is of about the fourth or fifth, as compared with the other Al lines. The assigned wave-lengths are probably correct to .3 or .4 of an Ångstrom unit. It is hardly possible that these lines are due to impurities; since elements which have lines near these positions have other stronger lines elsewhere which did not appear on our plates and the lines of the elements which are present as impurities are faint.

While these lines are apparently not to be placed in any of the simple series, there are however some interesting numerical relations:

I.

\begin{tabular}{|c|c|c|}
\hline$\lambda$ & $\sqrt{ }$ & Remarks. \\
\hline $\begin{array}{l}\text { 1. }\left\{\begin{array}{l}2907.4 \\
4150.6\end{array}\right. \\
\text { 2. }\left\{\begin{array}{l}3082.3 \\
4513.0\end{array}\right.\end{array}$ & $\begin{array}{l}34,394.97 \\
24,092.90 \\
10,302.07 \\
32,443.3 \\
22,158.2 \\
10,285.1\end{array}$ & $\begin{array}{c}\text { A new line } \\
\text { A new line } \\
\text { Frequency difference } \\
(2, p)-(3, d) \text { an arc line } \\
\text { An enhanced line } \\
\text { Frequency difference }\end{array}$ \\
\hline
\end{tabular}

Although the frequency differences between these two pairs are almost equal, it is difficult to see why the arc line $\lambda 3082$, which belongs to the first subordinate series, should be linked with the enhanced line $\lambda{ }_{45} \mathrm{I} 3$.

\section{II.}

I. The frequency of the line at 4I 50.6 is closely represented by the equation

$$
\begin{aligned}
& v=\left(2, p_{2}, \pi_{2}\right)-\mathrm{B}(\mathrm{I})-\left(3, p_{2}, \pi_{2}\right) \\
& v=24,066.32 \text { calculated } \\
& v=24,092.9 \text { observed. }
\end{aligned}
$$

${ }^{1}$ Astrophysical Journal, 52, p. 47, 1920. 